\title{
Friendly Process of Human-Computer Interaction - A Prototype System in Nostalgic World
}

\author{
Seiko Myojin ${ }^{1}$, Mie Nakatani ${ }^{2}$, Hirokazu Kato ${ }^{1}$, and Shogo Nishida ${ }^{1}$ \\ ${ }^{1}$ Graduate School of Engineering Science \\ Osaka University \\ 1-3, Machikaneyama, Toyonaka, Osaka, 560-8531, Japan \\ seiko@nishilab.sys.es.osaka-u.ac.jp, \\ \{kato, nishida\} asys.es.osaka-u.ac.jp \\ ${ }^{2}$ Center for the Study of Communication-Design \\ Osaka University \\ 1-1, Senri Expo Park, Suita, Osaka, 565-0826, Japan \\ mie@cscd.osaka-u.ac.jp
}

\begin{abstract}
In the interactive model considered herein, a user can be made to feel that the virtual world exists as tangibly as the real world does. Once "Friendly Process of Human-Computer Interaction" launches, a user can wait to see what happens before he or she takes action. Even if a user does nothing, the program still runs. A PC user is an audience. The computer program performs for its user audience. If the user is interested in the computer program's behavior, that user interacts with the PC as its audience. The purpose of our work was to examine the potentiality and significance of our interactive model. We integrated our concepts into our interactive design. In order to evaluate our concepts, we prepared three interactive movie system prototypes. The creation of our prototypes is well underway toward effectuating our concepts. After we improve our prototypes, we will then need to evaluate them.
\end{abstract}

Keywords: entertainment, emergent relationships, nostalgia, image recognition.

\section{Introduction}

Originally, the personal computer (hereinafter, "PC") was a business tool used for tasks such as document preparation or formula manipulation. A user commands the PC to manipulate a formula. A PC tells the user to push a button. This relationship was not an interactive one that elicited emotions or excitement, especially among those who had not worked with PCs before. In recent years, however, PC use has spread within the general public and now includes use as an entertainment tool. Everyone can enjoy telecasts or movies whenever and wherever they want. People can take pleasure in music by watching a live concert. Users can have fun chatting or playing Web camera games. Everywhere in contemporary society, you can find integrated circuit chips and sensors. People have no consciousness that they are being watched by computers. The systems that are not even regarded by users as computers 
have also increased. In the future, the relationship between users and computers will become even more varied.

We would like to propose herein an interactive model that is different from the traditional paradigm of a user's PC operations. In general, when a user does nothing, a PC will not start. If users want to avail themselves of that PC, they have to take necessary actions. Our concept offers a viewpoint differing from that of the traditional paradigm of a user operating a PC. With our design, characters from a virtual world will sometimes be able to suddenly tempt a real world user. However, this temptation does not beckon a user directly into a virtual world. A computer program performs for its user. If that user is interested in the computer program's behavior, the user can then interact with the computer as its audience. Even if no one is interacting with a PC, that PC still runs automatically. To the best of the authors' knowledge, no research has yet been carried out to discuss the potentiality and significance of such a relationship between a user and a PC. In the interactive model that we consider herein, a user can be made to feel that the virtual world exists, just like the real world. A user can wait to see what happens and what a PC thinks before that user takes action. Even if a user does nothing, the PC still starts. The PC user is an audience. A computer program performs for its user. If a user is interested in the computer program's behavior, that user interacts with the PC as its audience.

The purpose of our work described herein was to examine the potentiality and significance of our interactive model. We created original concepts and integrated them into our interactive design. In order to evaluate our concepts, we prepared three prototypical interactive movies, that is, three systems. The first system contains the minimum requirements necessary to bring our concepts to life as an interactive movie. The second system has the same function as the first system but an added feature is that, with the second system, a user can easily notice being recognized by that system. The third system uses a Web camera to recognize its user's motions. The creation of these prototype systems is well underway toward effectuating our concepts. In this paper, Chapter 2 discusses our concepts; Chapter 3 details the methods for realizing our concepts; Chapter 4 is an explanation of our prototype; Chapter 5 is devoted to a discussion about our prototype and related work; and, finally, Chapter 6 offers conclusions about this paper.

\section{Our Concept}

Our concept takes a viewpoint different from that of the traditional paradigm of a user's PC operation. We recognize a user before the user is able to notice that the PC recognizes him or her. A PC is also an invitation to the virtual world. A user faces the PC as an audience. A PC can prompt a user to think any of the following: "Maybe the PC recognizes me," "It is possible that the PC is responding to my actions," or "Perhaps I am a character in a story."

\subsection{A User May Notice That He or She Is Recognized in the Virtual World}

We consider the circumstances of using a PC. In general, a user realizes he or she can operate a number of characters and tools within a game before that user begins to play 
the game. However, in our work, a user does not know anything before entering into game play. The user has not even noticed that the game exists. For example, our scenarios look like circumstances from "The NeverEnding Story." Pastian, the protagonist, becomes aware that he is being recognized by the characters from the book that he is reading. We believe that if we can give PC users such awareness, we can then easily tempt them into the virtual world. Research on conventional entertainment systems shows the virtual world has increasingly grown in scale such that a user can be absorbed into it [1]. For example, CAVE [2] surrounds a user. It stimulates the user's five senses. We have tempted a user into the virtual world before he or she has noticed that even being recognized. The user can unconsciously open the door to the virtual world. As a result, that user can be absorbed into the virtual world.

\subsection{A User May Sense What a PC Thinks}

In general, there is always some button to push in response to a PC's command. After that, the $\mathrm{PC}$ reacts as the user would expect in response to that particular button. In this type of interaction, the PC is in control of its user. This type of interaction facilitates such programs as shooting games. We consider the interaction where a user becomes an observer. In daily life, such interactions regularly take place. Sometimes a person does not react to another person as expected. This interaction has interest. For example, your friends do not always react as you expect when you speak with them. People cannot necessarily reach clear understanding about each other. However, a person can certainly imagine what a friend thinks. Then many people are interested in that interaction. Now replace this interaction between the two friends with an interaction between a user and a PC. Although a PC does not always react to a user as expected, such unexpected responses can lead to new and interesting pathways.

\subsection{Emergent Relationships}

If a user can feel the virtual world to exist as surely as the real world does, we can more easily tempt him or her into the virtual world. To make this happen, the characters in the virtual world and the PC user must have some kind of relationship. For example, in "The NeverEnding Story," there was a queen awaiting a child's help. That child was Pastian. Pastian sensed this relationship between himself and that of the world of Fantargen. Pastian came to feel that the world of "Fantargen," the world found inside the book that the boy was reading, existed as surely as the real world did. We call this phenomenon "Emergent Relations," and we have incorporated this idea into our interface.

\section{Our Methods}

We took our original concepts and incorporated them into interactive designs. In order to evaluate our concepts, we prepared three prototypical interactive movies. The theme of these movies is nostalgia. For purposes of our discussion, "nostalgia" refers to a person remembering the good old days in a sentimental way. Nostalgia does not usually require that a person consciously summon up a nostalgic feeling. Rather, 
nostalgia is an emotion that spontaneously springs up in someone's heart as if a person suddenly crooned a favorite song without any reason. This is why nostalgia is a suitable theme to the application of our concepts and prototype production. Our movies could be something that provokes people into remembering their fond memories.

Our concept consists of three elements. First, a user may notice that he or she is recognized in the virtual world. Second, a user might sense what a PC is thinking. As a result of this second element, a user can wait to see what happens next, what their PC thinks, before that user takes any action. The third element is that of the emergent relationship. A user will feel that the virtual world exists as tangibly as the real world does. We have constructed our movies prototypes in order to effectuate the interface of these elements on a PC. We have done so for the following reasons: When a user does nothing, the PC can play our movie as if it were a screen saver playing. We can stimulate the user's awareness with our timing. We can also instigate interactions between a user and a population of characters and tools from within the virtual world. Furthermore, the virtual world characters and the user can develop a relationship.

We considered what movie features we should use to bring our concepts to life. We drew up a movie plan in which we project the characters' lines and narration both with sound and captions. Our movies are played from the perspective of the main character. Our movie regards a user as one of the characters within the movie. Even if a user does not interact with the movie characters, our stories would be designed to develop and finish automatically. In the characters' lines, our movies do not explicitly invite a user inside the virtual world by such exhortations as Join with us or Write on it. Rather, our movies express the characters' lines such that a user can somehow sense meaning from the context. For example, the user senses that he or she is a character within the movie by hearing or seeing dialogue such as We were waiting for you to finish writing it. By using a Web camera, our system activates when it recognizes a user in front of the screen. The challenge is to keep the user from noticing this. In the virtual world, multiplicities of characters are acting. By coordinating the virtual world timing, our system is able to recognize a user as one of the virtual world characters and the story develops in response to the user's appearance. The user does not necessarily notice that he or she is recognized by a Web camera. However, even if a user does notice the active Web camera, the user may still be interested in the characters' behavior and wish to interact with them. Nonetheless, the user does not yet know how to interact. The user needs to figure out how to interact. It is not difficult for users to learn how to interact. Our movie provides a number of clues.

Other points to note are as follows: In selecting the descriptions, the camera work, and the colors, we took note of what images might be remembered from scenery in the past. For example, we did not use realistic colors within the world of memories. Even if the picture is different from the true look of past scenery, it is still important to induce a nostalgic texture. For the keywords in the characters' lines, we set keywords that the average adult will not often use. In order to do this, we chose childlike expressions and youthful words. For our landscape, we used an ordinary school building and its adjacent park so that a user could easily relate to the scenery. We integrated sound effects to our benefit: the sounds of someone turning a page or footsteps, the noise of a crowd, a cicada's buzz, and other such sounds. We have 
attached importance to realistic sounds. The effectiveness of sound to induce a user's emotions and imagery is discussed in Reference [3], below. We considered the length of time of our movies. Our movies need enough time to make a user notice that he or she is being recognized by our system. They require sufficient time to interact. Our movies should appear the way a screen saver does, that is, disappearing while a user is wondering about the story. Our movies make a user understand a story in a short time. Therefore, when a user does not interact with our movie, that movie plays no longer than approximately five minutes. If a user does interact with our movie, we then play our movie for a maximum of about ten minutes.

\section{The Prototype}

\subsection{Prototype Architecture}

Fig. 1 shows our prototype. We used Windows XP and Macromedia Flash (Action Script 2.0). In Graduation2, we also employed Microsoft Visual Studio.NET $2003 \quad$ (Microsoft Development Environment 2003, .NET Framework 1.1, Visual C\#.NET), and put "Vidgets[4]" to use as a library for

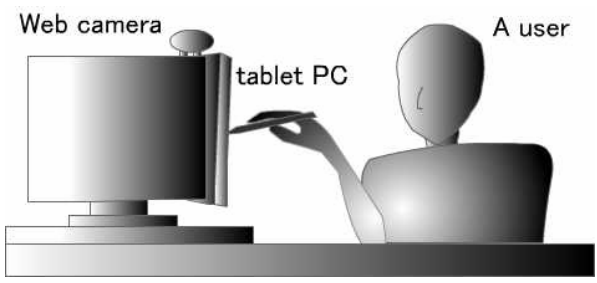

Fig. 1. Architecture of our prototype image recognition. Our Web camera's specifications are as follows: The minimallength subject distance is $20 \mathrm{~cm}$; the video resolution is $640 \times 480$ with a CMOS sensor.

\subsection{Movie Scenarios}

Graduation1 (Fig. 2): The theme of this movie is a memory illusion about graduation. Students in Japan usually buy their school yearbook when they graduate from school. A school yearbook is those students' photograph collection. Students and teachers tend to write messages for their friends or students in these yearbooks. The protagonist of this movie visits his or her old school. The protagonist graduated from the school about ten years ago. He or she walks into classroom. After a while the protagonist meets past classmates. Then strange things happen there.

Graduation2 (Fig. 3): Although the scenario of this movie is identical to that

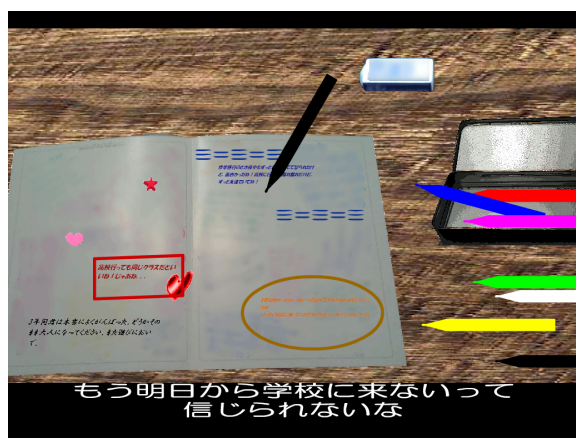

Fig. 2. Graduation1 of Graduation1, this movie serves another function, which is to show random noise. The user is unable to view the movie clearly because of the random noise whenever the user's face gets too near to the screen. This is inspired by the idea of a dream 
state. If a person is having a pleasant dream, they try to sleep not to wake. However, their visibilities in the dreams become poor because they almost start to wake up.

DARUMA-san1 (Fig. 4): This movie's theme is a memory illusion about "DARUMA-san ga Koronda." "DARUMA-san ga Koronda" is a kind of tag game, one that is the traditional play of Japanese children in a group. Children choose one child to be called "Oni." Oni has to catch other children. Oni turns around and faces a tree or wall. While Oni counts for ten seconds, the other children can scatter in all directions. After Oni has finished counting to ten, he or she says "DA RU MA - SA N GA KO RO N DA" and turns around, looking. Other children can draw closer to Oni provided that Oni does not see them. However, if a moving child is seen by Oni, Oni can then catch

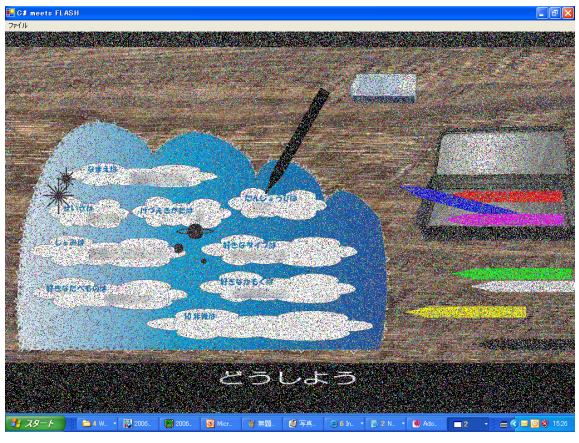

Fig. 3. Graduation2

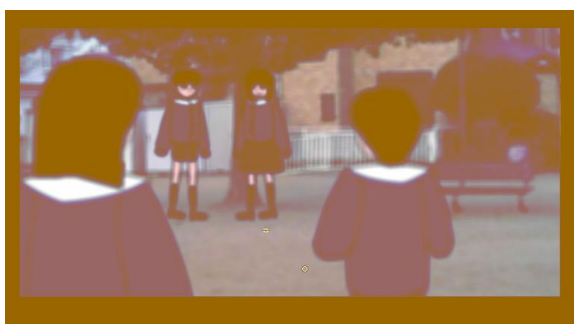

Fig. 4. DARUMA-san 1 him or her. If Oni manages to catch all the children, Oni wins the game. In summary, Oni calls out "DA RU MA - SA N GA KO RO N DA" and then looks around to try and catch any moving children. If a child touches Oni without Oni's seeing their approach, then all the children get a free pass to scatter from Oni in any direction. Although there are other rules at play in various regions of Japan, this is the general method of "DARUMA-san ga Koronda".

\subsection{Future Work}

The following parameters sum up our prototypes: Each of our interactive movies includes three specific functions designed to effectuate our concepts. (1) We project the characters' lines and narration both through sound and caption; (2) Our movie incorporates the user as one of the characters within the movie; and (3) Even if a user does not interact with the other characters in our movie, the movie will nonetheless have to develop and finish automatically. The first movie system contains the minimum requirements to bring our concepts to life as an interactive movie. The second movie system serves the same function as the first system but, additionally, a user can easily observe being recognized by the system. Finally, the third movie system utilizes a Web camera to recognize the motions of its user. Although we have nearly completed our work, the systems still have some shortcomings. We will continue to improve our systems. We tested Graduation1 with one user. We observed that Graduation1 succeeded in getting the user to notice that she was a character 
within the movie. She could find almost how to interact. Once we have improved our prototypes, we will then need to evaluate our systems more thoroughly.

\section{Related Works}

Our concepts resemble Kohei Asano's "Shiawase wa soko ni aru[5]." Asano is in the Engineering Department at Tokyo Polytechnic University. In Asano's system, when a user waves a hand at a cat, the cat has some kind of reaction. A small camera incorporated into the display recognizes the motion of the user's hand and the system responds by playing a randomized video of cats. Cats are naturally capricious. Humans understand that a cat does not always react to a person's specific action. This is why, even if the system plays a randomized video of cats, a user still feels that he or she has interacted with a cat. Asano's system is predicated upon new content, rather than a new interactive model.

Although there are only a few journal articles that focus on inducing nostalgia, there are many that address works of art. For example, "Imagery Box"[5], "Interval"[5] "Kioku"[6]. Although the user may simply enjoy seeing such work, he or she is unable to feel becoming or being a character within the story. These projects are not interactive movies.

Toshio Iwai's "ElectroPlankton[7]" contains similarities to our project's concepts. In "ElectroPlankton," users do not know how to interact before they enter into the game. However, the characters in "ElectroPlankton" do not engage in any relationship with users. In that regard, our concepts differ from those of "ElectroPlankton."

\section{Conclusions}

In the interactive model that we have considered, a user can be made to feel that the virtual world exists as tangibly as the real world does. A user can wait to see what happens and what a PC thinks before he or she takes action. Even if a user does nothing, the PC still starts. A user is a PC's audience. A computer program performs for its user. If a user is interested in the computer program's behavior, then that user will interact with the PC and be its audience. The purpose of our work was to examine the potentiality and significance of our interactive model.

We have created original concepts and incorporated them within our interactive design. In order to evaluate our concepts, we prepared three prototypical interactive movie systems. The first system contains the minimum requirements to effectuate our concepts as an interactive movie. The second system serves the same function as the first system but also allows a user to easily notice that he or she is being recognized by the system. The third system utilizes a Web camera to recognize the motion of users. The creation of these prototypes is well underway toward effectuating our concepts. After we improve our prototypes, we will then need to evaluate them. In our evaluative experiment, we may have users download our software. Alternatively, we might choose a sparsely populated street, set up some screens there on the street, and then play our movie upon one of those screens. On the other screens, we would play a conventional movie. Afterward, we would conduct interviews with users to obtain those users' impressions. 


\section{References}

1. Bowman, D.A., Raja, D., Lucas, J., Datey, A.: Exploring the Benefits of Immersion for Information Visualization, HCII2005 (2005)

2. Cruz-Neira, C., Sandin, D.J., DeFanti, T.A.: Surround-Screen Projection-Based Virtual Reality: The Design and Implementation of the CAVE. In: Proc.of SIGGRAPH'93, pp. 135-142 (1993)

3. Berman, A.D., Cruz-Neira, C., Berman, S.L., Tuch, L.: 9/11: Building A World of Survivor Memories, HCII2005 (2005)

4. Vidgets: http://qwik.jp/media2005/Vidgets.html (Tateo Igarashi uses Vidgets in his lecture Media Information Science) (in Japanese)

5. NHK: Digital Stadium (in Japanese), http://www.nhk.or.jp/digista/

6. TBS: DigiCon6+2 (in Japanese) (2006), http://www.tbs.co.jp/digicon/digicon/

7. Iwai, T.: ElectroPlankton, (in Japanese) http://www.watch.impress.co.jp/game/docs/ 20050408/ele.htm 